\title{
Pain relief procedures before high-dose-rate brachytherapy for non-surgical treatment of cervix
}

\section{cancer}

\author{
Antonio Cássio Assis Pellizzon, MD, PhD \\ Department of Radiation Oncology, AC Camargo Cancer Center, Sao Paulo, Brazil
}

\begin{abstract}
Cervical cancer $(\mathrm{CC})$ is a neoplasm with great potential for prevention, but it is still an important public health problem in most developing countries. No significant difference is found in the literature between intracavitary high-doserate (HDR) and low-dose-rate (LDR) brachytherapy, when considering overall, disease specific, and recurrence-free survivals. Cervical dilatation is mandatory for the insertion of intra-uterine tandems for CC intracavitary brachytherapy. Pain and discomfort may eventually be the limiting factors of the procedure, sometimes leading to unsatisfactory results in terms of adequate position of the applicator set. In this paper, we critically reviewed the current sedation and anesthetic options for comfort and safety procedures when performing intracavitary brachytherapy.

J Contemp Brachytherapy 2018; 10, 6: 567-569 DOI: https://doi.org/10.5114/jcb.2018.81027
\end{abstract}

Key words: brachytherapy, cervix cancer, intracavitary, gynecological cancer, pain relief.

\section{Purpose}

Cervical cancer (CC) is a neoplasm with a great potential for prevention, but it is still an important public health problem in most developing countries, leading to a significant number of deaths in young women (15-50 years old). In 2012, there has been a total of 528,000 new diagnosis and 266,000 deaths due to CC worldwide, accounting for $7.5 \%$ of all deaths from female cancers [1].

The treatment of choice for patients with locally advanced CC is definitive radiotherapy combined with chemotherapy, including intracavitary brachytherapy. One published meta-analysis showed no significant differences between low-dose-rate brachytherapy (LDR) and high-dose-rate (HDR) brachytherapy when considering overall, disease specific, and recurrence-free survivals. Local control, recurrence, metastasis incidence rates, and treatment-related complications were neither different when comparing both techniques [2].

Most radiation oncology departments or centers usually have at least one dedicated HDR unit, with a significant number of patients treated as outpatients but the presence of an associated anesthetic team is not frequent.

For HDR intracavitary technique (iHDR), one of the most difficult and painful procedures is the cervical dilatation, which is necessary for the insertion of the intra-uterine tandem. Despite the thinner diameter of the tandems used in iHDR (compared to LDR tandems), dif- ferent degrees of discomfort are experienced by the patients, even when anti-spasmodic treatment is prescribed before the procedure.

Using the terms "sedation; anesthesia; gynecological brachytherapy", we performed a search in the Pubmed and found 14 publications. Three reports about associated pain and discomfort similar to iHDR were also analyzed.

\section{Discomfort and pain}

The discomfort experienced by patients during iHDR is a combination of causes and the explications for the discomfort during iHDR are multiple. Some regions of the female reproductive tract, such as the isthmus and myometrium, have a great number of nerve endings [3]. The cervix and uterus are insensitive to heat or fine touch stimuli, but dilatation of cervix and uterine distension causes pain. The dilatation and stimulation of these areas can modify the intensity of the symptoms at certain moments of the procedure, especially during the introduction of intrauterine tandem [4]. Indeed, the pain and discomfort may eventually be the limiting factors of the outpatient procedure, sometimes leading to unsatisfactory results in terms of adequate position of the applicator set.

The presence of tandem in the body of the uterus stimulates sympathetic autonomic afferents, which enter the spinal cord at the thoracic and lumbar levels, causing poorly localized central and lower abdominal pain, 
that can be accompanied by nausea and vomiting, while the distention of the cervix and upper vagina stimulates parasympathetic autonomic afferents from the splanchnic sacral nerves, that results in low back pain. The distention of the entire vagina by packing can also stimulate the pudendal nerves increasing the discomfort [5]. There may also be further delayed pain caused by the release of prostaglandins [6].

There is a paucity of data and prospective randomized clinical trials to evaluate the relations of iHDR and pain or discomfort, but it could be associated with the same discomfort related to hysteroscopy. A recent meta-analysis published by Ahmad et al. showed no benefits for the use of oral opioids and/or oral non-steroidal anti-inflammatory drugs prior or during these procedures. Secondary adverse events were also evaluated is this publication and divided into three categories: vasovagal reactions, non-pelvic pain, and allergic reactions. For all three sub-groups, there was no significant difference demonstrated between the local intervention and control groups [7].

\section{Anxiety}

Anxiety is widely recognized as side effect of gynecologic procedures, and there is no agreement on the point of maximal physical and emotional distress. In general, nulliparous women, postmenopausal patients, women with a history of dysmenorrhea, or suffering from anxiety are more likely to experience greater pain during gynecologic procedures. For anxious patients, it is suggested to include oral anxiolytic medication and, if possible, a dedicated emotional support of a person [8]. Visual or auditory distraction should be considered in all instances, even when using analgesic conscious maintenance [9].

\section{Local interventions}

\section{Local anesthesia}

The use of local spray of lidocaine was evaluated by Chen et al. and, although safe, it was considered not effective in pain relief [10].

Paracervical block of the cervix is another form of local anesthesia. A systematic review of the various local anesthetic techniques used during outpatient hysteroscopy concluded that paracervical local anesthetic injections are a reasonable method for patients who undergo such procedures, but there are still doubts if it is appropriate to perform paracervical injections for cancer patients. There is an evidence of benefit for the use of local anesthetics for outpatient hysteroscopy and sonohysterography, but local anesthetics may be just considered for procedures lasting no more than 30 minutes, as no significant beneficial effect of its use was identified after this period [11].

\section{Cervical dilators}

The use of cervical dilators, such as osmotic ones (synthetic laminaria) has being described in the literature. The osmotic dilator has to be inserted into the cervix 10 to 12 hours before each iHDR and removed just before the pro- cedure [12]. The use of associated sedation (opioids and/ or paracervical block) is optional.

\section{Oral medications}

Some drugs can be used as an analgesic or sedative. Diclofenac sodium, pentazocine hydrochloride, and morphine hydrochloride hydrate are the analgesics most frequently used. Hydroxyzine hydrochloride or haloperidol were described to be administered as a sedative in some cases [5].

The administration of misoprostol $400 \mu \mathrm{g}$ before tandem insertion was evaluated by Cepni et al. in a randomized trial. After 80 patients with evaluated CC, they concluded that the medication taken orally 3 hours before tandem application facilitates the procedure and increases patient's tolerability and comfort [13].

\section{Conscious sedation}

Bhanabhai et al. reported their conscious sedation protocol using an intravenous opioid (morphine, hydromorphone, or fentanyl) in addition to intravenous midazolam, noting that the use of fentanyl rather than hydromorphone or morphine did not result in a significant decrease in pain during the procedure [14].

According to Kwekkeboom et al., $26 \%$ of patients who underwent iHDR experienced severe uterine pain even with conscious sedation [9].

Leong et al. reported their experience with combined interstitial and iHDR. The anesthetic regimen consisted of a combination of intravenous midazolam, propofol, fentanyl, oxycodone, and local anesthesia applied to the vaginal canal and a paracervical block was considered safe, effective, and reproducible [15].

The short recovery time presented with conscious sedation protocols shows its applicability to outpatient treatment, and the American Brachytherapy Society recommends that conscious sedation should be used during iHDR insertions whenever possible [16].

\section{Anesthetic procedures}

Lumbar epidural anesthesia and spinal anesthesia are suitable for inpatients. Some institutions advise its use also for outpatients; however, there is a paucity of data in literature reporting these procedures as well as the time until discharge. Complications associated with epidural anesthesia include local anesthetic-induced toxicity, dural puncture, hematoma, and infection at the site of puncture [5]. Epidural anesthesia is wildly used in US and Europe but is underused in the rest of the world due to the shortage of anesthesiologists.

\section{Visual analogue scale}

A recent systematic review to be published showed that one of the most commonly and effectively used form of pain assessment is the visual analogue scale (VAS). This scale, among others, is an important instrument that assists the medical staff involved in the procedure in the interpretation of pain, besides allowing the visualization 
of the evolution of the patient during the proposed treatment in a more reliable way. It is easily applicable and safe tool that could be useful to analyze effectiveness of the analgesic procedures, which procedures have had better results as well as detection of any deficiency in the treatment, according to the degree of improvement or worsening of the pain [17].

\section{Conclusions}

The implementation of sedation protocols might result in better positioning and/or packing, despite no consensus found in the literature regarding the optimal method of pain relief for iHDR. Methods that facilitate the adequate, quick, and safe administration of analgesic or anesthetic drugs that reduce the pain and discomfort experienced by CC patients during iHDR are essential and required. Only a small number of studies have compared the efficacy or toxicity of the various anesthetic methods that can be used, therefore, it is a wide and open field for scientific investigations.

Evidence for some interventions exists; however, the interpretation of intervention comparisons is limited by the use of different regimens, pain measurement scales, patient populations, and procedure techniques. Each method of analgesia and sedation have advantages and disadvantages, and the indication of one or other must be balanced and individualized.

\section{Disclosure}

Author reports no conflict of interest.

\section{References}

1. Ferlay J, Soerjomataram I, Dikshit R et al. Cancer incidence and mortality worldwide: sources, methods and major patterns in GLOBOCAN2012. Int J Cancer 2015; 136: E359-386.

2. Liu R, Wang $X$, Tian JH et al. High dose rate versus low dose rate intracavity brachytherapy for locally advanced uterine cervix cancer. Cochrane Database Syst Rev 2014; 10: CD007563.

3. Yunker A, Curlin H, Banet N et al. Does the uterine cervix become abnormally reinnervated after subtotal hysterectomy and what is the association with future trachelectomy? J Minim Invasive Gynecol 2015; 22: 261-267.

4. Pellizzon ACA. Braquiterapia de alta taxa de dose: tumores ginecológicos. In: Pellizzon ACA et al. Rotinas e condutas em radioterapia. $3^{\text {th }}$ ed. Lemar, São Paulo 2008; 80-87.

5. Smith MD. Analgesia for pelvic brachytherapy. $\mathrm{Br} J$ Anaesth 2002; 88: 270-276

6. Farrugia M, Hussain SY. Hysteroscopic endometrial ablation using the Hydro ThermAblator in an outpatient hysteroscopy clinic: feasibility and acceptability. J Minim Invasive Gynecol 2006; 13: 178-182.

7. Ahmad G, Attarbashi S, O'Flynn H, Watson AJ. Pain relief in office gynaecology: a systematic review and meta-analysis. Eur J Obstet Gynecol Reprod Biol 2011; 155: 3-13.

8. Kamer S, Ozsaran Z, Celik O et al. Evaluation of anxiety levels during intracavitary brachytherapy applications in women with gynecological malignancies. Eur J Gynaecol Oncol 2007; 28: 121-124.

9. Kwekkeboom KL, Dendaas NR, Straub Metal. Patterns of pain and distress during high-dose-rate intracavity brachytherapy for cervical cancer. J Support Oncol 2009; 7: 108-114.
10. Chen HC, Leung SW, Wang CJ et al. Local vaginal anesthesia during high-dose-rate intracavitary brachytherapy for cervical cancer. Int J Radiat Oncol Biol Phys 1998; 42: 541-544.

11. Cooper NA, Khan KS, Clark TJ. Local anaesthesia for pain control during outpatient hysteroscopy: systematic review and meta-analysis. BMJ 2010; 340: c1130.

12. Mayr NA, Sorosky JI, Zhen W et al. The use of laminarias for osmotic dilation of the cervix in gynecological brachytherapy applications. Int I Radiat Oncol Biol Phys 1998; 42: 1049-1053.

13. Cepni K, Gul S, Cepni I et al. Randomized trial of oral misoprostol treatment for cervical ripening before tandem application in cervix cancer. Int J Radiat Oncol Biol Phys 2011; 81: 778-781.

14. Bhanabhai H, Samant R, Grenier L et al. Pain assessment during conscious sedation for cervical cancer high-dose-rate brachytherapy. Curr Oncol 2013; 20: e307-310.

15. Leong YH, Tan KHS, Choo BA et al. Novel anesthetic technique for combined intracavitary and interstitial brachytherapy for cervix cancer in an outpatient setting. J Contemp Brachytherapy 2017; 9: 236-241.

16. Nag S, Erickson B, Thomadsen B et al. The American Brachytherapy Society recommendations for high-dose-rate brachytherapy for carcinoma of the cervix. Int J Radiat Oncol Biol Phys 2000; 48: 201-211.

17. Chiarotto A, Maxwell LJ, Ostelo RW et al. Measurement properties of Visual Analogue Scale, Numeric Rating Scale and Pain Severity subscale of the Brief Pain Inventory in patients with low back pain: a systematic review. J Pain 2018: S1526-5900(18)30400-0. 